Review

\title{
Review on Techniques for Thermal Characterization of Graphene and Related 2D Materials
}

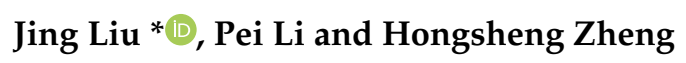 \\ College of New Materials and New Energies, Shenzhen Technology University, Shenzhen 518116, China; \\ 2070413005@stumail.sztu.edu.cn (P.L.); 20183280058@stumail.sztu.edu.cn (H.Z.) \\ * Correspondence: liujing@sztu.edu.cn
}

Citation: Liu, J.; Li, P.; Zheng, H. Review on Techniques for Thermal Characterization of Graphene and Related 2D Materials. Nanomaterials 2021, 11, 2787. https://doi.org/ 10.3390/nano11112787

Academic Editor: Marco Cannas

Received: 6 September 2021

Accepted: 19 October 2021

Published: 21 October 2021

Publisher's Note: MDPI stays neutral with regard to jurisdictional claims in published maps and institutional affiliations.

\begin{abstract}
The discovery of graphene and its analog, such as $\mathrm{MoS}_{2}$, has boosted research. The thermal transport in 2D materials gains much of the interest, especially when graphene has high thermal conductivity. However, the thermal properties of 2D materials obtained from experiments have large discrepancies. For example, the thermal conductivity of single layer suspended graphene obtained by experiments spans over a large range: $1100-5000 \mathrm{~W} / \mathrm{m} \cdot \mathrm{K}$. Apart from the different graphene quality in experiments, the thermal characterization methods play an important role in the observed large deviation of experimental data. Here we provide a critical review of the widely used thermal characterization techniques: the optothermal Raman technique and the micro-bridge method. The critical issues in the two methods are carefully revised and discussed in great depth. Furthermore, improvements in Raman-based techniques to investigate the energy transport in 2D materials are discussed.
\end{abstract}

Keywords: optothermal Raman technique; thermal transport; 2D materials

\section{Introduction}

Since the discovery of graphene and other $2 \mathrm{D}$ materials such as $\mathrm{MoS}_{2}$, various properties of 2D materials have been intensively studied [1-5]. The ultra-high thermal conductivity of graphene has led to extensive experimental research and theoretical simulations about the energy transport in it in past decades [3,6,7]. The thermal transport in other 2D materials also gains much interest for its promising applications [8,9]. However, compared with the thermal conductivity $(\kappa)$ obtained from simulations, $\kappa$ obtained from experiments shows large discrepancies. For example, $\kappa$ of suspended single layer graphene (SLG) ranges from 1100 to $5300 \mathrm{~W} / \mathrm{m} \cdot \mathrm{K}[3,10]$, depending on the thermal characterization method and the fabrication method of graphene. $\kappa$ of supported graphene drops to hundreds $\mathrm{W} / \mathrm{m} \cdot \mathrm{K}$, which is also related to the substrate $[6,11,12]$. It is well accepted that $\kappa$ of supported graphene is suppressed due to phonon leakage $[6,13]$. Table 1 summarizes $\kappa$ of suspended and supported graphene by different experimental methods. A large discrepancy among $\mathcal{K}$ can be observed. This discrepancy arises from the characterization methods and the quality of the graphene. The thermal characterization techniques of $2 \mathrm{D}$ materials include the optothermal Raman technique [3,11,14], micro-bridge method [6,9], time-domain thermoreflectance (TDTR) [15] and Johnson noise thermometry [16]. In this paper, we will focus on the optothermal Raman technique and the micro-bridge method. The critical issues faced in the above two methods will be discussed in depth. The issues in the optothermal Raman technique include the accuracy of the laser power absorbed by 2D materials, stress effect and inter-phonon branch nonequilibrium. When it comes to the micro-bridge method, the thermal resistance of the 2D materials should be properly chosen to guarantee measurement accuracy. These issues undermine the measurement accuracy in thermal transport characterization of 2D materials [17]. 
Table 1. Thermal conductivity of graphene obtained by experiments.

\begin{tabular}{cccc}
\hline $\boldsymbol{\kappa}(\mathbf{W} / \mathbf{m} \cdot \mathbf{K})$ & Method & Brief Description & References \\
\hline$\sim 3000-5000$ & Raman optothermal & Suspended SLG ${ }^{1}$, exfoliated & {$[3]$} \\
\hline $2500+1100 /-1050$ & Raman optothermal & Suspended SLG, CVD ${ }^{2}$ & {$[11]$} \\
\hline $400-1800$ & Raman optothermal & $\begin{array}{c}\text { Suspended SLG with crystal } \\
\text { lattice defects }\end{array}$ & {$[18]$} \\
\hline $730-880 \pm 60$ & Micro-bridge & $\begin{array}{c}\text { Suspended bilayer graphene, } \\
\text { PMMA }^{3} \text { residues on the surface }\end{array}$ & {$[19]$} \\
\hline $1896 \pm 390$ & Raman optothermal & Suspended bilayer graphene & {$[20]$} \\
\hline 365 & Transient & Supported SLG on PMMA, giant \\
thermoelectrical & technique (TET) & [12] & \\
\hline $370+650 /-320$ & Raman optothermal & Supported SLG on copper, CVD & {$[11]$} \\
\hline 600 & Micro-bridge & $\begin{array}{c}\text { Supported SLG on amorphous } \\
\text { SiO }{ }_{2}^{4}, \text { exfoliated }\end{array}$ & {$[6]$} \\
\hline
\end{tabular}

${ }^{1}$ SLG: Single layer graphene. ${ }^{2}$ CVD: Chemical vapor deposition. ${ }^{3}$ PMMA: Polymethyl methacrylate. ${ }^{4} \mathrm{SiO}_{2}$ : Silicon dioxide.

\section{Raman Optothermal Method}

The characteristic peaks in Raman spectra of the 2D materials have strong temperature dependence. It is possible to make use of the Raman spectra to characterize the thermal transport in 2D materials [8,21-24]. Balandin first developed the confocal Raman spectroscopy to measure $\kappa$ of suspended graphene [3]. Schematic of the optothermal Raman technique is shown in Figure 1. As the laser spot is much smaller than the suspended graphene size, the heat is propagating radially to the edges. By obtaining the Raman shift temperature coefficient $\left(\chi_{T}\right)$ and the Raman shift power coefficient $\left(\chi_{P}\right)$ of $G$ peak, the thermal conductivity of graphene can be expressed as $\kappa=\chi_{T}(L / 2 h W) / \chi_{P}$ [3]. Here, $L, h$ and $W$ are the distance from the hot spot to the heat sink, thickness of SLG and width of the sample, respectively. The optothermal Raman technique is proven to be powerful and widely used in characterizing the energy transport in 2D materials [25]. Advantages of the optothermal Raman method include minimal sample preparation, high spatial resolution and material specificity [17]. However, it is important to point out that several critical issues should be carefully considered regarding the utilization of the Raman optothermal method.

One important parameter in the deduction of $\mathcal{K}$ of graphene is the laser power $(P)$ absorbed by the graphene. Usually, two methods are employed to obtain $P$. One is calculating the absorbed power based on the optical properties [3]. It is well accepted that the absorption coefficient $\left(\alpha_{G}\right)$ of SLG is $2.3 \%$ [2]. Thus, $P$ can be described as $P=I_{0} A\left(1-\exp \left(-\alpha_{G} \delta\right)\right)$, where $I_{0}$ is the laser intensity on the surface, $A$ is the illuminated area and $\delta$ is the thickness of SLG [3]. However, the $\alpha_{G}$ is easily affected by many factors, such as the wrinkles and the strain [26]. The optical properties can vary greatly from sample to sample, resulting in uncertainty in the laser absorption. In supported graphene, the laser absorption is significantly affected by the interface-induced optical interference [27], which leads to great uncertainty in the laser absorption calculation. Another method is directly measuring the transmitted power. Thus, the absorbed power can be obtained by subtracting the transmitted power from the total incident laser power. However, a very small proportion of the incident laser power is absorbed by graphene. Thus, even very little variation in the transmitted power can lead to great uncertainty in the absorbed power. The uncertainty in $P$ will affect the accuracy of $\chi_{P}$, which further introduces uncertainty into the derivation of $\kappa$. It is difficult to determine the uncertainty of $P$. If there is $10 \%$ uncertainty in $\chi_{P}, 10 \%$ uncertainty will be introduced to $\kappa$. 


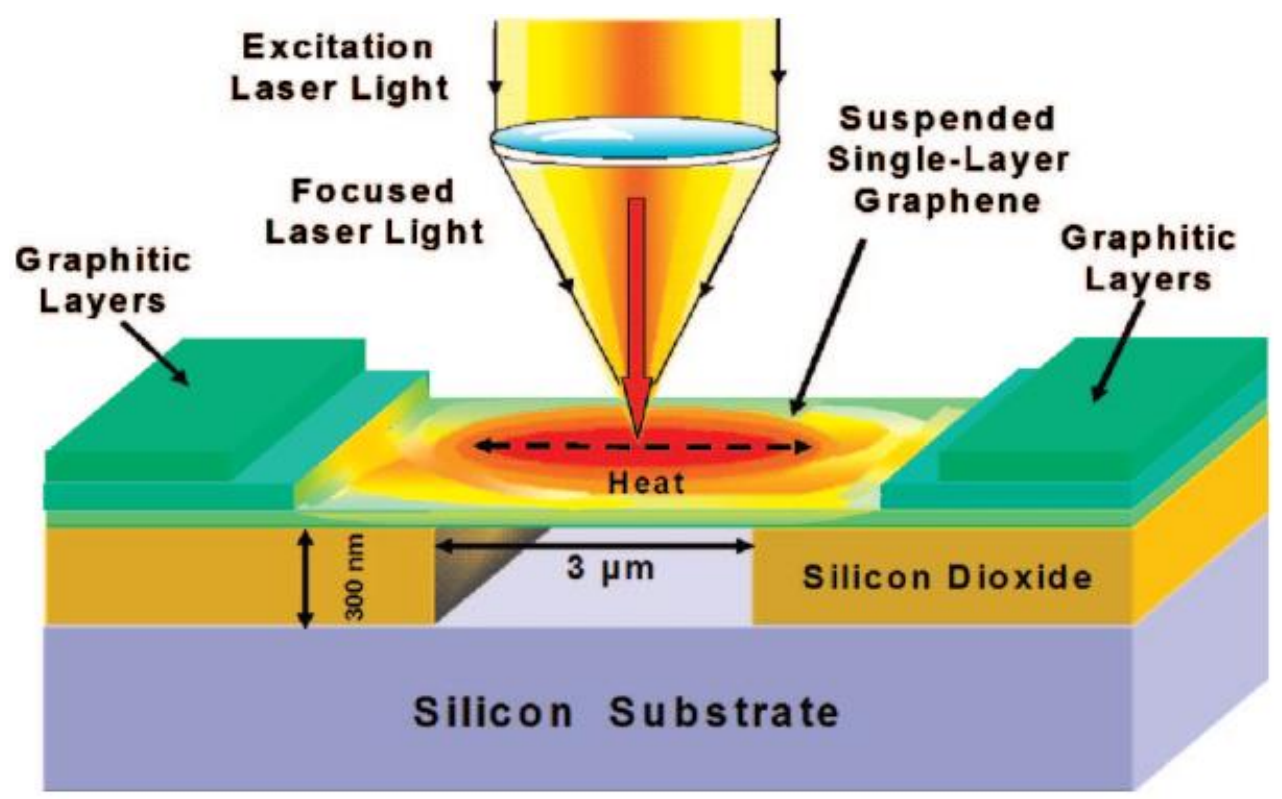

Figure 1. Schematic of the optothermal Raman technique. Reprinted with permission from ref. [3]. Copyright 2008 American Chemical Society.

Another source of uncertainty in the optothermal Raman method is the stress effect in the graphene. During the temperature coefficient calibration, the whole sample is in thermal equilibrium. However, the graphene experiences thermal nonequilibrium in the experiment. This leads to the different stress effects in the graphene during the calibration process and experiment. Thus, the temperature probed by Raman spectroscopy is not precise, which further introduces uncertainty into $\kappa$ determination [28]. Apart from this, the Raman spectroscopy actually detects the temperature of the optical phonons, which is easily affected by the thermomechanical stress. The thermomechanical stress in few layers graphene (FLG) alters the interatomic-potential, which affects the energy of the optical phonons [17]. Theoretical simulation shows that the uncertainty caused by the thermomechanical stress in $\mathcal{K}$ of FLG can be higher than 20\% [17].

It is critically important to point out that the Raman optothermal method is based on an assumption that different phonon branches are in thermal equilibrium under the photon excitation. However, Ruan et al., first reported that the phonon branches were in strong thermal nonequilibrium by employing the density functional perturbation theory (DFPT) [29]. For example, the steady-state temperature of transverse optical phonons $\left(T_{T O}\right)$ can be $14.8 \%$ higher than that $\left(T_{Z A}\right)$ of out-of-plane acoustic $(Z A)$ phonons at the center of the SLG [30]. By using the multitemperature model (MTM) developed by Ruan et al., the predicted $\kappa$ of SLG is increased by $67 \%$ [30]. Ruan's theoretical simulation enlightened the experimental work about the thermal nonequilibrium among phonon branches. The temperature differences between different phonon branches in 2D materials under Raman excitation were first verified and detected by Wang's group by experiment [31]. Wang et al., distinguished the temperatures of optical $(O P)$ and acoustic $(A P)$ phonons under phonon excitation in 2D materials by constructing steady and nanosecond (ns) inter-phonon branch energy transport states [31]. By developing the nanosecond energy transport state-resolved Raman (ns ET-Raman) technique, the temperature difference $\left(\triangle T_{O P-A P}\right)$ between $O P$ and $A P$ is reported to be $30 \%$ larger than the Raman-probed temperature rise in $\mathrm{MoS}_{2}$ [31].

In most research about the energy transport in 2D materials by Raman spectroscopy, the hot carrier diffusion effect is not considered, which is more prominent as the laser spot size is smaller than $0.5 \mu \mathrm{m}$ [28]. Here, we look at a $\mathrm{MoS}_{2} / \mathrm{c}-\mathrm{Si}$ structure and discuss what will happen after the laser illumination on the sample. Figure 2a shows the physical principle of the electrons and holes diffusion under the laser illumination. Subsequent to laser irradiation, the electrons and holes are generated by absorbing photons. In extremely 
short time ( $\sim \mathrm{ps})$, the excess energy $\left(\Delta E=E-E_{g}\right.$ ) of the electrons will quickly dissipate to other unexcited electrons and the lattice. Then, the electrons and holes (hot carriers) diffuse and recombine, releasing the energy by scattering with the optical phonons. This leads to a much larger thermal source area than the excitation spot. The specific process is described in detail in the reference [32]. The electron-hole diffusion has a negligible effect on the thermal conduction in suspended 2D materials [33]. However, the effect of the hot carrier diffusion should be carefully handled when determining the interface thermal energy transport between graphene and substrate.
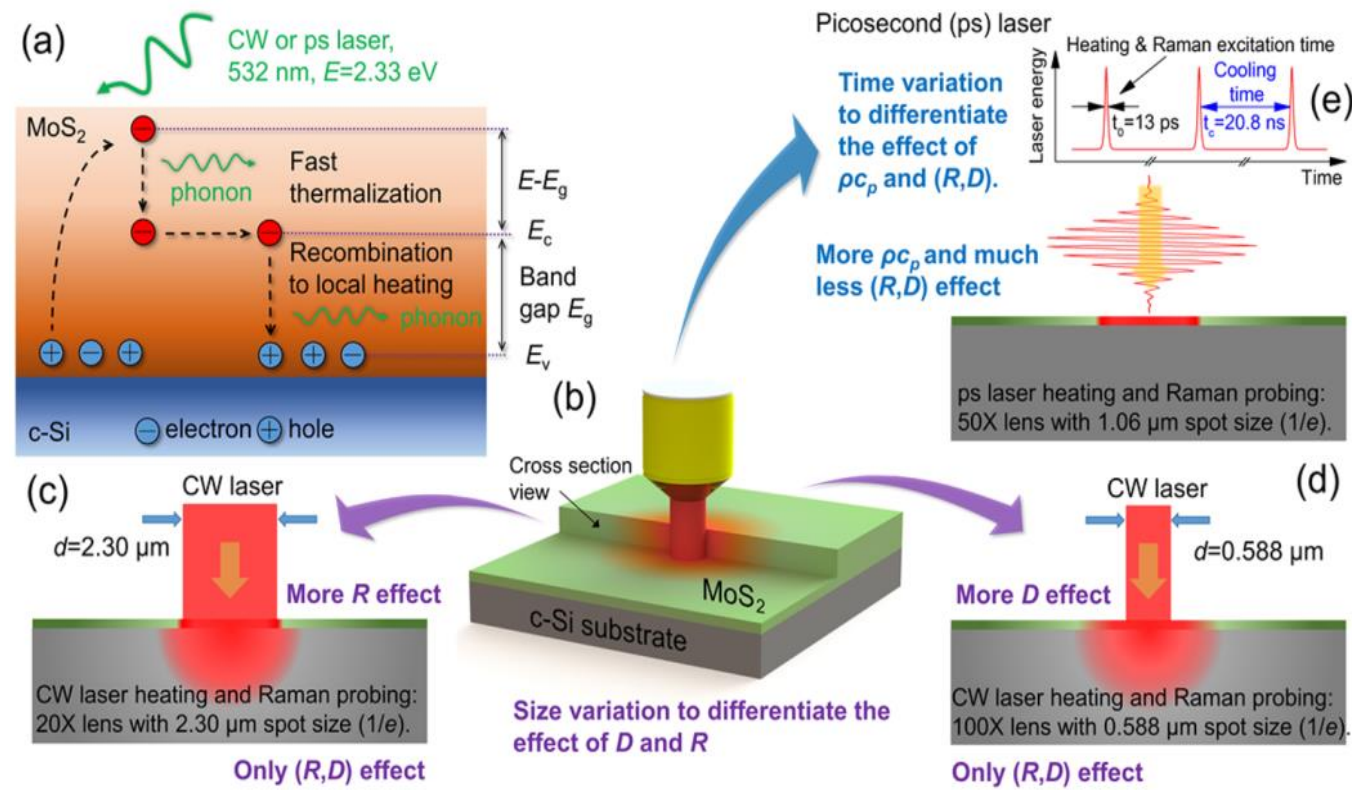

Figure 2. (a) Hot carrier diffusion in $\mathrm{MoS}_{2} / \mathrm{c}-\mathrm{Si}$ under laser illumination (not to scale): $E_{v}$ and $E_{c}$ are the valence band and conduction band, respectively, $E_{g}$ is the bandgap of $\mathrm{MoS}_{2}, E$ is the photon energy of the incident laser; (b) schematic of the experiment setup (not to scale): $\rho c_{p}$ is the volumetric heat capacity of the sample; $(\mathbf{c}, \mathbf{d})$ continuous wave $(\mathrm{CW})$ laser is used to heat the sample under $20 \times$ and $100 \times$ objective lens to achieve different hot aera size: $R$ and $D$ are the interface thermal resistance and hot carrier diffusion coefficient, respectively; (e) picosecond pulsed laser is used under $50 \times$ objective lens to achieve zero thermal transport state. Reprinted with permission from ref. [27]. Copyright 2017 American Chemical Society.

\section{Micro-Bridge Method}

Shi Li et al., first employed the micro-bridge method to measure $\kappa$ of graphene supported on amorphous $\mathrm{SiO}_{2}$ [6]. The schematic of the experiment is shown in Figure 3. There are four $\mathrm{Au} / \mathrm{Cr}$ resistance thermometer (RT) lines in the setup. The two straight RT lines (RT2 and RT3) cover the two ends of the graphene. The U-shaped RT1 and RT4 separate from both the graphene and the RT2 and RT3. During the experiment, the RT1 is self-heated by applying current into it. Based on the thermal resistance circuit shown in Figure 3c, thermal resistance $\left(R_{S}\right)$ of the central beam including both graphene and $\mathrm{SiO}_{2}$ can be expressed as: $R_{s}=R_{b} \frac{\Delta T_{2, m}-\Delta T_{3, m}}{\Delta T_{3, m}+\Delta T_{4, m}}[6]$. Here, $\Delta T_{j, m}(j=2,3,4)$ is the temperature rise at the middle point of RT. $R_{b}$ is the thermal resistance of each RT line with the $\mathrm{SiO}_{2}$ beam. By measuring the Rs before and after removing the graphene, $\kappa$ of graphene is determined. The accuracy of the micro-bridge method is guaranteed only as $R_{s}$ is comparable to $R_{b}$. As $\kappa$ of the graphene is low or the length of the graphene $/ \mathrm{SiO}_{2}$ beam is longer than tens of $\mu \mathrm{m}$, $R_{s}$ will be much larger than $R_{b}$. Thus, the heat conducting into the graphene $/ \mathrm{SiO}_{2}$ beam will be very small, which will lead to great uncertainty in the measurement of $\Delta T_{m}$. This will further increase the uncertainty in the determination of $\kappa$ of graphene. Overall, the micro-bridge method is feasible in principle, but its measurement accuracy is guaranteed 
when the sample has proper thermal resistance. In addition, it is technically challenging and time consuming to fabricate the whole measurement device.
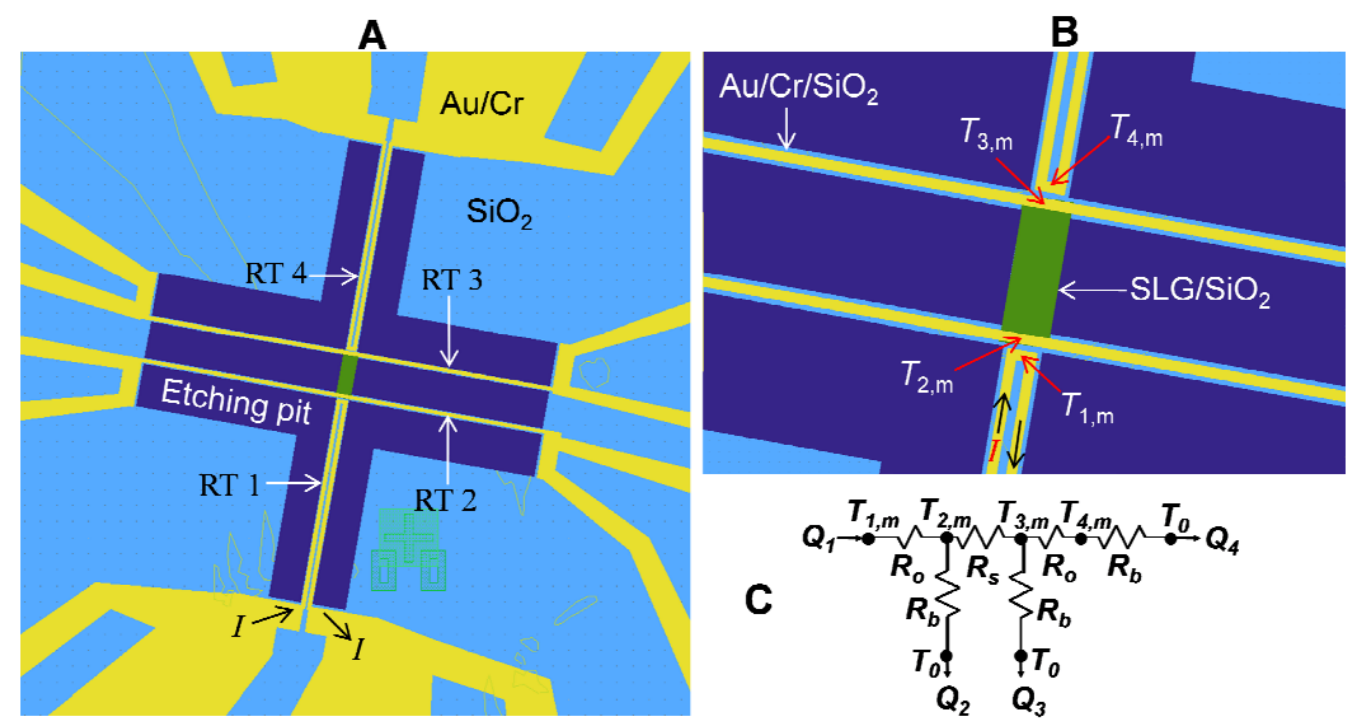

Figure 3. (A,B) SEM images of micro-bridge method setup; (C) circuit of the thermal resistance. Reprinted with permission from ref. [6]. Copyright 2010 American Association for the Advancement of Science.

The sample size tested by the Raman spectroscopy method and micro-bridge method is at the level of several $\mu \mathrm{m}$. However, the mean free path (MFP) of phonons in graphene can be as long as hundreds of micrometers [34], surpassing the sample size. This will result in a strong phonon-edge scattering effect in the graphene. To investigate the intrinsic $\mathcal{K}$ of graphene without or with minimal phonon-edge scattering, Liu et al., first developed the differential transient electrothermal technique (TET) to characterize $\kappa$ of giant graphene supported by polymethyl methacrylate (PMMA) [12]. The experiment setup is shown in Figure 4. The graphene supported by PMMA is suspended between two electrodes. During the experiment, the whole sample is fed through with step current to induce Joule heating in it. The voltage evolution $(V(t))$ of the sample is recorded by an oscilloscope. The thermal diffusivity $(\beta)$ of the whole sample can be obtained by fitting the $V(t) \sim t$ curve. $\kappa_{\text {eff }}$ of the whole sample is calculated as $\kappa_{\text {eff }}=\beta \cdot \rho c_{p}$. Through simulation, it is found that the interface thermal resistance between graphene and PMMA is negligible in $\kappa_{\text {eff. }}$. Thus, $\kappa_{\text {eff }}$ can be written as $\kappa_{\text {eff }}=f\left(\kappa_{p}, \kappa_{g}, \delta_{p}, \delta_{g}\right)$. Here, the subscripts $p$ and $g$ indicate PMMA and graphene, respectively. $\delta$ is thickness. $\mathcal{K}$ of SLG supported by PMMA was determined to be $365 \mathrm{~W} / \mathrm{m} \cdot \mathrm{K}$ [12], which is only $60 \%$ of $\kappa$ of graphene supported by amorphous $\mathrm{SiO}_{2}$ [6]. The authors attributed the low thermal conductivity of SLG on PMMA to abundant carbon atoms in the PMMA [12]. The abundant carbon atoms lead to a strong phonon scattering effect between SLG and PMMA. 


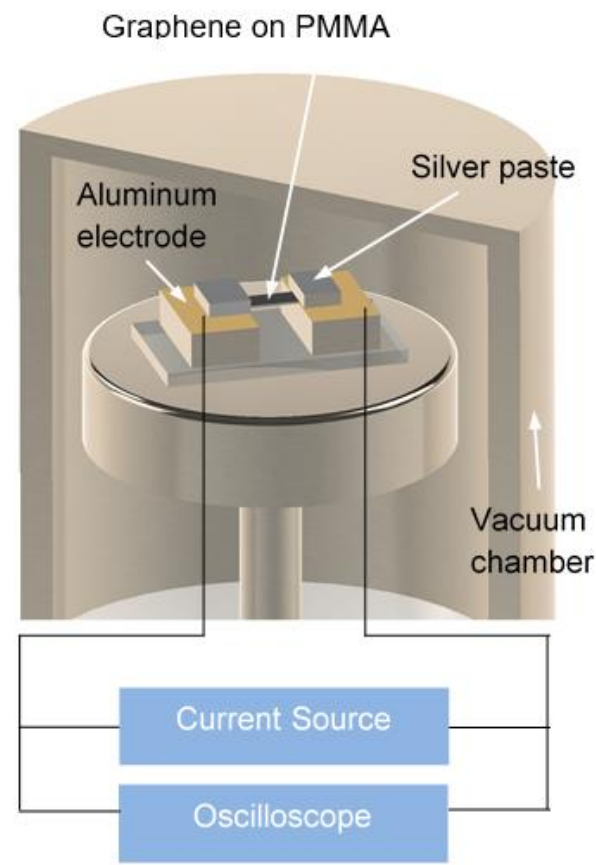

Figure 4. Experiment setup of the TET technique. Reprinted from ref. [12].

\section{Improvements in the Optothermal Raman Technique}

In order to resolve the challenges mentioned above in the optothermal Raman technique, various Raman-based techniques, including frequency-domain energy transport state-resolved Raman (FET-Raman) [35,36] and energy transport state-resolved Raman (ET-Raman) [33], were developed. They can free the Raman thermometry from the laser absorption and the temperature coefficient calibration. In the FET-Raman experiment, the 2D material experiences two energy transport states. The first one is the steady-state heating induced by a CW laser. By varying the incident laser power $(P)$, the Raman shift power coefficient (RSC) $\chi_{\text {steady-state }}=\partial \omega / \partial P=\alpha \cdot(\partial \omega / \partial T) \cdot f_{1}(\kappa)$ is obtained. Here, $\alpha$ is the laser absorption coefficient, $\partial \omega / \partial T$ is the Raman shift temperature coefficient and $\kappa$ is the in-plane thermal conductivity. The second energy transport state is the transient-state heating induced by a square wave-modulated CW laser. Similarly, an RSC can be obtained: $\chi_{\text {transient }}=\partial \omega / \partial P=\alpha \cdot(\partial \omega / \partial T) \cdot f_{2}\left(\kappa, \rho c_{p}\right)$, where $\rho c_{p}$ is the volumetric heat capacity of the sample. Since the thermal diffusion lengths in two energy states are different, a normalized RSC parameter can be defined as $\Theta=\chi_{\text {transient }} / \chi_{\text {steady-state }}=f_{3}\left(\kappa, \rho c_{p}\right)$ [35]. Thus, the effect of $\alpha$ and $\partial \omega / \partial T$ is eliminated. By interpolating the $\Theta$ obtained from the experiment into the $\Theta-\kappa$ curve obtained from a 3D numerical simulation of the $\mathrm{MoSe}_{2}$ sample, $\kappa$ of $\mathrm{MoSe}_{2}$ is determined. The feasibility of the FET-Raman technique has been verified by employing the FET-Raman to determine $\kappa$ of $\mathrm{MoSe}_{2}$ and the anisotropic thermal conductivities of carbon fibers $[35,36]$.

Inspired by the theoretical simulation by Ruan et al. [29,30,37], Wang et al., first developed ns ET-Raman to explore the inter-phonon branch non-equilibrium effect in 2D materials under photon excitation [31]. Under CW laser excitation, the local temperature rise $\left(\Delta T_{m}\right)$ consists of the temperature rise of acoustic phonons $\left(\Delta T_{A P}\right)$ and the temperature difference between optical phonons and acoustic phonons $\left(\Delta T_{O A}\right) . \Delta T_{m}$ can be expressed as:

$$
\Delta T_{m}=\Delta T_{A P}+\Delta T_{O A}=\Delta T_{A P}+\delta I / G_{p p}
$$

Here, $I$ is the intensity of the absorbed laser at location $r . \delta(0<\delta<1)$ is the portion of the laser energy transfer from optical phonons $(O P)$ to acoustic phonons $(A P)$. $G_{p p}$ is 
the coupling factor between $O P$ and $A P$. Furthermore, the Raman intensity weighted temperature rise $\left(\left.\Delta \bar{T}_{m}\right|_{C W}\right)$ probed by the Raman spectroscopy can be expressed as [21]:

$$
\begin{gathered}
\left.\Delta \bar{T}_{m}\right|_{C W}=\frac{\iint \Delta T_{m} I_{C W} e^{-z / \tau_{L}} 2 \pi r d r d z}{\iint c e^{e^{-z / \tau}} 2 \pi r d r d z} \\
=\left.\Delta \bar{T}_{O A}\right|_{C W}+\left.\Delta \bar{T}_{A P}\right|_{C W}=\frac{1}{3} \times \frac{I_{0}}{\tau_{L}} \times \frac{\delta}{\left.G_{p p}\right|_{C W}}+\left.\Delta \bar{T}_{A P}\right|_{C W}
\end{gathered}
$$

Here, $I_{C W}$ is the laser intensity distribution of the $C W$ laser. $\left.\Delta \bar{T}_{O A}\right|_{C W}$ and $\left.\Delta \bar{T}_{A P}\right|_{C W}$ are the Raman intensity weighted temperature difference between $O P$ and $A P$ and the temperature rise of the acoustic phonons, respectively. $I_{0}$ is the absorbed laser power per unit area, and $\tau_{L}$ is the absorption depth. Before determining the percentages of $\left.\Delta \bar{T}_{O A}\right|_{C W}$ and $\left.\Delta \bar{T}_{A P}\right|_{C W}$ in $\left.\Delta \bar{T}_{m}\right|_{C W},\left.G_{p p}\right|_{C W}$ should be figured out first by experiment. By constructing a $3 \mathrm{D}$ heat conduction model for a $55 \mathrm{~nm}$-thick $\mathrm{MoS}_{2},\left.\Delta \bar{T}_{A P}\right|_{C W}$ is obtained as $\left.\Delta \bar{T}_{A P}\right|_{C W}=0.94+2.86 e^{-1.65 r_{0}} . r_{0}$ is the radius of the laser spot. The Raman shift is proportional to the temperature rise caused by unit power; thus, the Raman shift coefficient $\chi_{C W}$ can be expressed as [31]:

$$
\chi_{C W}=A \times\left[\left(0.94+2.86 e^{-1.65 r_{0}}+\frac{1}{3} \times \frac{P}{\pi r_{0}^{2} \tau_{L}} \times \frac{\delta}{\left.G_{p p}\right|_{C W}}\right)\right] / P
$$

Here, $A$ is a constant. In the experiment, three objective lenses $(20 \times, 50 \times$ and $100 \times)$ are employed to obtain the $\chi_{C W}-r_{0}$ relationship. By fitting $\chi_{C W}-r_{0}$ relationship with Equation (3), $\left.G_{p p}\right|_{C W}$ can be determined. Furthermore, the Raman intensity weighted temperature rise $\left.\Delta \bar{T}_{O A}\right|_{C W}$ and $\left.\Delta \bar{T}_{A P}\right|_{C W}$ can be figured out through the $3 \mathrm{D}$ conduction numerical calculation. It is found that the temperature difference between $O P$ and $A P$ accounts for more than $30 \%$ of the temperature rise detected by Raman [31]. Zobeiri et al., further characterized the thermal nonequilibrium between $O P$ and $A P$ under photon excitation in graphene paper [38]. The Raman intensity weighted temperature rise of $O P$ is found to be $82.1 \%$ higher than that of $A P$ under $100 \times$ laser heating [38], which indicates the importance of taking interphonon thermal nonequilibrium effects into consideration in the optothermal Raman technique.

To consider the hot carrier diffusion effect in the thermal transport in 2D materials, Yuan et al., first developed the ET-Raman to determine the interface thermal resistance and hot carrier diffusion coefficient in $\mathrm{MoS}_{2}$ supported by c-Si $[27,33]$. The ET-Raman includes two energy transport states: the zero thermal transport state and the steady-state thermal transport. The zero thermal transport state is obtained by applying a picosecond pulsed laser under a $50 \times$ objective lens. In an extremely short pulse ( 13 ps) time, only the fast thermalization process happens, so the heat conduction in the lattice can be neglected. By varying the laser power, the RSC $\chi_{p s}=\partial \omega / \partial P$ is obtained, which is more affected by the $\rho c_{p}$ rather than by the hot carrier diffusion coefficient $(D)$ and interface thermal resistance $(R)$. In the steady-state thermal transport, the RSC under $20 \times$ and $100 \times$ objective lenses are obtained by applying the $C W$ laser. Both $\chi_{C W 20}$ under the $20 \times$ objective lens and $\chi_{C W 100}$ under the $100 \times$ objective lens carries the information of $D$ and $R$. However, $\chi_{C W 20}$ is more affected by $R$, while $\chi_{C W 100}$ is more affected by $D$. Here, the normalized RSC is defined as $\Theta_{1}=\chi_{C W 20} / \chi_{p s}$ and $\Theta_{2}=\chi_{C W 100} / \chi_{p s}$. By simulating a 3D heat conduction model in the sample, the RSC contour with $R$ and $D$ as variables is obtained. The cross point of the $\Theta_{1}$ curve and $\Theta_{2}$ curve gives the value of $R$ and $D$.

\section{Conclusions}

In summary, though facing several critical problems, the optothermal Raman technique and micro-bridge method still show suitability and feasibility in energy transport characterization in 2D materials. Much pioneering work about the thermal non-equilibrium in different phonon branches has been reported. However, the physical model and data fitting used in the pioneering work still suffer great uncertainties and make the study 
rather semi-quantitative. Secondly, past work has studied the thermal nonequilibrium in suspended 2D materials. However, for supported 2D materials, how the interface resistance between 2D material and substrate affects the $O P-A P$ thermal nonequilibrium is unclear. Further work can be focused on the OP-AP thermal nonequilibrium in supported $2 \mathrm{D}$ materials.

Author Contributions: J.L. conceived and wrote the review; P.L. and H.Z. cooperated in bibliographic searches, studies. J.L. conceived and revised the paper. All authors have read and agreed to the published version of the manuscript.

Funding: This research was funded by the Natural Science Foundation of Top Talent of SZTU (2019209) and the Guangdong Basic and Applied Basic Research Foundation (2020A1515110389).

Institutional Review Board Statement: Not applicable.

Informed Consent Statement: Not applicable.

Data Availability Statement: No new data were created or analyzed in this review. Data sharing is not applicable to this article.

Acknowledgments: J. Liu thanks Xinwei Wang of Iowa State University for the discussion.

Conflicts of Interest: The authors declare no conflict of interest.

\section{References}

1. Ahmadi, Z.; Yakupoglu, B.; Azam, N.; Elafandi, S.; Mahjouri-Samani, M. Self-limiting laser crystallization and direct writing of 2D materials. Int. J. Extreme Manuf. 2019, 1, 015001. [CrossRef]

2. Nair, R.R.; Blake, P.; Grigorenko, A.N.; Novoselov, K.; Booth, T.; Stauber, T.; Peres, N.M.R.; Geim, A.K. Fine structure constant defines visual transparency of graphene. Science 2008, 320, 1308. [CrossRef]

3. Balandin, A.A.; Ghosh, S.; Bao, W.; Calizo, I.; Teweldebrhan, D.; Miao, F.; Lau, C.N. Superior thermal conductivity of single-layer graphene. Nano Lett. 2008, 8, 902-907. [CrossRef]

4. Yoon, D.; Son, Y.-W.; Cheong, H. Negative thermal expansion coefficient of graphene measured by Raman spectroscopy. Nano Lett. 2011, 11, 3227-3231. [CrossRef]

5. Yoon, D.; Son, Y.-W.; Cheong, H. Strain-dependent splitting of the double-resonance Raman scattering band in graphene. Phys. Rev. Lett. 2011, 106, 155502. [CrossRef]

6. Seol, J.H.; Jo, I.; Moore, A.L.; Lindsay, L.; Aitken, Z.H.; Pettes, M.T.; Li, X.; Yao, Z.; Huang, R.; Broido, D.; et al. Two-dimensional phonon transport in supported graphene. Science 2010, 328, 213-216. [CrossRef]

7. Hopkins, P.E.; Baraket, M.; Barnat, E.V.; Beechem, T.E.; Kearney, S.P.; Duda, J.C.; Robinson, J.T.; Walton, S.G. Manipulating thermal conductance at metal-graphene contacts via chemical functionalization. Nano Lett. 2012, 12, 590-595. [CrossRef]

8. Wang, R.; Wang, T.; Zobeiri, H.; Yuan, P.; Deng, C.; Yue, Y.; Xu, S.; Wang, X. Measurement of the thermal conductivities of suspended $\mathrm{MoS}_{2}$ and $\mathrm{MoSe}_{2}$ by nanosecond ET-Raman without temperature calibration and laser absorption evaluation. Nanoscale 2018, 10, 23087-23102. [CrossRef]

9. Jo, I.; Pettes, M.T.; Kim, J.; Watanabe, K.; Taniguchi, T.; Yao, Z.; Shi, L. Thermal Conductivity and Phonon Transport in Suspended Few-Layer Hexagonal Boron Nitride. Nano Lett. 2013, 13, 550-554. [CrossRef] [PubMed]

10. Chen, S.; Li, Q.; Zhang, Q.; Qu, Y.; Ji, H.; Ruoff, R.S.; Cai, W. Thermal conductivity measurements of suspended graphene with and without wrinkles by micro-Raman mapping. Nanotechnology 2012, 23, 365701. [CrossRef]

11. Cai, W.; Moore, A.; Zhu, Y.; Li, X.; Chen, S.; Shi, L.; Ruoff, R.S. Thermal transport in suspended and supported monolayer graphene grown by chemical vapor deposition. Nano Lett. 2010, 10, 1645-1651. [CrossRef]

12. Liu, J.; Wang, T.; Xu, S.; Yuan, P.; Xu, X.; Wang, X. Thermal conductivity of giant mono- to few-layered CVD graphene supported on an organic substrate. Nanoscale 2016, 8, 10298-10309. [CrossRef]

13. Ong, Z.-Y.; Pop, E. Effect of substrate modes on thermal transport in supported graphene. Phys. Rev. B 2011, 84, 075471. [CrossRef]

14. Soini, M.C.; Zardo, I.; Uccelli, E.; Funk, S.; Koblmüller, G.; I Morral, A.F.; Abstreiter, G. Thermal conductivity of GaAs nanowires studied by micro-Raman spectroscopy combined with laser heating. Appl. Phys. Lett. 2010, 97, 263107. [CrossRef]

15. Jiang, P.; Qian, X.; Yang, R. Time-domain thermoreflectance (TDTR) measurements of anisotropic thermal conductivity using a variable spot size approach. Rev. Sci. Instrum. 2017, 88, 074901. [CrossRef]

16. Waissman, J.; Anderson, L.E.; Talanov, A.V.; Yan, Z.; Shin, Y.J.; Najafabadi, D.H.; Taniguchi, T.; Watanabe, K.; Skinner, B.; Matveev, K.A.; et al. Measurement of Electronic Thermal Conductance in Low-Dimensional Materials with Graphene Nonlocal Noise Thermometry. arXiv 2021, arXiv:2101.01737. Available online: https: / /arxiv.org/abs/2101.01737 (accessed on 10 October 2021).

17. Beechem, T.; Yates, L.; Graham, S. Invited Review Article: Error and uncertainty in Raman thermal conductivity measurements. Rev. Sci. Instrum. 2015, 86, 041101. [CrossRef] 
18. Malekpour, H.; Ramnani, P.; Srinivasan, S.; Balasubramanian, G.; Nika, D.L.; Mulchandani, A.; Lake, R.K.; Balandin, A.A. Thermal conductivity of graphene with defects induced by electron beam irradiation. Nanoscale 2016, 8, 14608-14616. [CrossRef]

19. Jo, I.; Pettes, M.T.; Lindsay, L.; Ou, E.; Weathers, A.; Moore, A.L.; Yao, Z.; Shi, L. Reexamination of basal plane thermal conductivity of suspended graphene samples measured by electro-thermal micro-bridge methods. AIP Adv. 2015, 5, 053206. [CrossRef]

20. Li, H.; Ying, H.; Chen, X.; Nika, D.L.; Cocemasov, A.I.; Cai, W.; Balandin, A.A.; Chen, S. Thermal conductivity of twisted bilayer graphene. Nanoscale 2014, 6, 13402-13408. [CrossRef] [PubMed]

21. Calizo, I.; Balandin, A.A.; Bao, W.; Miao, F.; Lau, C.N. Temperature Dependence of the Raman Spectra of Graphene and Graphene Multilayers. Nano Lett. 2007, 7, 2645-2649. [CrossRef] [PubMed]

22. Wang, T.; Liu, J.; Xu, B.; Wang, R.; Yuan, P.; Han, M.; Xu, S.; Xie, Y.; Wu, Y.; Wang, X. Identifying the Crystalline Orientation of Black Phosphorus by Using Optothermal Raman Spectroscopy. ChemPhysChem 2017, 18, 2828-2834. [CrossRef] [PubMed]

23. Wang, T.; Han, M.; Wang, R.; Yuan, P.; Xu, S.; Wang, X. Characterization of anisotropic thermal conductivity of suspended nm-thick black phosphorus with frequency-resolved Raman spectroscopy. J. Appl. Phys. 2018, 123, 145104. [CrossRef]

24. Sahoo, S.; Gaur, A.P.S.; Ahmadi, M.; Guinel, M.J.-F.; Katiyar, R.S. Temperature-Dependent Raman Studies and Thermal Conductivity of Few-Layer MoS 2 . J. Phys. Chem. C 2013, 117, 9042-9047. [CrossRef]

25. Malekpour, H.; Balandin, A.A. Raman-based technique for measuring thermal conductivity of graphene and related materials. J. Raman Spectrosc. 2017, 49, 106-120. [CrossRef]

26. Ni, G.-X.; Yang, H.-Z.; Ji, W.; Baeck, S.-J.; Toh, C.-T.; Ahn, J.-H.; Pereira, V.M.; Özyilmaz, B. Tuning Optical Conductivity of Large-Scale CVD Graphene by Strain Engineering. Adv. Mater. 2013, 26, 1081-1086. [CrossRef]

27. Yuan, P.; Wang, R.; Tan, H.; Wang, T.; Wang, X. Energy Transport State Resolved Raman for Probing Interface Energy Transport and Hot Carrier Diffusion in Few-Layered $\mathrm{MoS}_{2}$. ACS Photon. 2017, 4, 3115-3129. [CrossRef]

28. Wang, R.; Wang, T.; Zobeiri, H.; Li, D.; Wang, X. Energy and Charge Transport in 2D Atomic Layer Materials: Raman-Based Characterization. Nanomaterials 2020, 10, 1807. [CrossRef]

29. Vallabhaneni, A.K.; Singh, D.; Bao, H.; Murthy, J.; Ruan, X. Reliability of Raman measurements of thermal conductivity of single-layer graphene due to selective electron-phonon coupling: A first-principles study. Phys. Rev. B 2016, 93, 125432. [CrossRef]

30. Lu, Z.; Vallabhaneni, A.; Cao, B.; Ruan, X. Phonon branch-resolved electron-phonon coupling and the multitemperature model. Phys. Rev. B 2018, 98, 134309. [CrossRef]

31. Wang, R.; Zobeiri, H.; Xie, Y.; Wang, X.; Zhang, X.; Yue, Y. Distinguishing Optical and Acoustic Phonon Temperatures and Their Energy Coupling Factor under Photon Excitation in nm 2D Materials. Adv. Sci. 2020, 7, 2000097. [CrossRef]

32. Yuan, P.; Liu, J.; Wang, R.; Wang, X. The hot carrier diffusion coefficient of sub-10 nm virgin MoS $_{2}$ : Uncovered by non-contact optical probing. Nanoscale 2017, 9, 6808-6820. [CrossRef]

33. Yuan, P.; Tan, H.; Wang, R.; Wang, T.; Wang, X. Very fast hot carrier diffusion in unconstrained MoS $_{2}$ on a glass substrate: Discovered by picosecond ET-Raman. RSC Adv. 2018, 8, 12767-12778. [CrossRef]

34. Fugallo, G.; Cepellotti, A.; Paulatto, L.; Lazzeri, M.; Marzari, N.; Mauri, F. Thermal Conductivity of Graphene and Graphite: Collective Excitations and Mean Free Paths. Nano Lett. 2014, 14, 6109-6114. [CrossRef]

35. Zobeiri, H.; Wang, R.; Wang, T.; Lin, H.; Deng, C.; Wang, X. Frequency-domain energy transport state-resolved Raman for measuring the thermal conductivity of suspended nm-thick MoSe 2 . Int. J. Heat Mass Transf. 2019, 133, 1074-1085. [CrossRef]

36. Wang, R.; Zobeiri, H.; Lin, H.; Qu, W.; Bai, X.; Deng, C.; Wang, X. Anisotropic thermal conductivities and structure in lignin-based microscale carbon fibers. Carbon 2019, 147, 58-69. [CrossRef]

37. Sullivan, S.; Vallabhaneni, A.; Kholmanov, I.; Ruan, X.; Murthy, J.; Shi, L. Optical Generation and Detection of Local Nonequilibrium Phonons in Suspended Graphene. Nano Lett. 2017, 17, 2049-2056. [CrossRef] [PubMed]

38. Zobeiri, H.; Hunter, N.; Wang, R.; Wang, T.; Wang, X. Direct Characterization of Thermal Nonequilibrium between Optical and Acoustic Phonons in Graphene Paper under Photon Excitation. Adv. Sci. 2021, 8, 2004712. [CrossRef] 